\title{
Vessel wall signal enhancement on 3-T MRI in acute stroke patients after stent retriever thrombectomy
}

\author{
Peter Abraham, BA, J. Scott Pannell, MD, David R. Santiago-Dieppa, MD, Vincent Cheung, MD, \\ Jeffrey Steinberg, MD, Arvin Wali, BA, Mihir Gupta, MD, Robert C. Rennert, MD, \\ Roland R. Lee, MD, and Alexander A. Khalessi, MD, MS
}

Department of Neurosurgery, University of California, San Diego, California

\begin{abstract}
OBJECTIVE In vivo and in vitro studies have demonstrated histological evidence of iatrogenic endothelial injury after stent retriever thrombectomy. However, noncontrast vessel wall (VW)-MRI is insufficient to demonstrate vessel injury. Authors of this study prospectively evaluated iatrogenic endothelial damage after stent retriever thrombectomy in humans by utilizing high-resolution contrast-enhanced VW-MRI. Characterization of VW-MRI changes in vessels subject to mechanical injury from thrombectomy may allow better understanding of the biological effects of this intervention.
\end{abstract}

METHODS The authors prospectively recruited 11 patients for this study. The treatment group included 6 postthrombectomy patients and the control group included 5 subjects undergoing MRI for nonvascular indications. All subjects were evaluated on a Signa HD× 3.0-T MRI scanner with an 8-channel head coil. Both pre- and postcontrast T1-weighted Cube VW images as well as MR angiograms were acquired. Sequences obtained for evaluation of the brain parenchyma included diffusion-weighted, gradient echo, and T2-FLAIR imaging. Two independent neuroradiologists, who were blinded to the treatment status of each patient, determined the presence of VW enhancement. Patient age, National Institutes of Health Stroke Scale score on presentation, location of occlusion, stroke etiology, type of device used, number of device deployments, Thrombolysis in Cerebral Infarction (TICl) reperfusion score, stroke volume, and 90-day modified Rankin Scale score were also noted.

RESULTS Postcontrast T1-weighted VW enhancement was detected in the $\mathrm{M}_{2}$ segment in $100 \%$ of the thrombectomy patients, in the $M_{1}$ segment in $83 \%$, and in the internal carotid artery in $50 \%$. One patient also demonstrated $A_{1}$ segment enhancement, which was attributable to thrombectomy treatment of that vessel segment during the same procedure. None of the control patients demonstrated VW enhancement of their intracranial vasculature on T1-weighted images.

CONCLUSIONS The study findings suggest that VW injury incurred during stent retriever thrombectomy can be reliably detected utilizing contrast-enhanced 3-T VW-MRI. The results further demonstrate that endothelial injury is associated with oversizing of stent retrievers relative to the treated vessel. Further studies are needed to evaluate the clinical significance of endothelial injury and to characterize the differential effects of various devices.

https://thejns.org/doi/abs/10.3171/2017.1.FOCUS16492

KEY WORDS magnetic resonance imaging; stroke; thrombectomy; vessel wall signal enhancement; stent retriever

$\mathrm{E}$ NDOVASCULAR thrombectomy for the treatment of acute ischemic stroke has demonstrated dramatic benefit in patients with large-vessel occlusions. However, a subset of patients still demonstrates poor clinical outcomes despite complete angiographic revascularization. ${ }^{10}$ This discordance between angiographic results and clinical status is particularly evident in early, as com- pared with late, thrombectomy trials..$^{3,6}$ It is thought that iatrogenic disruption of the endothelium during thrombectomy ${ }^{2,4}$ may initiate a cascade of events leading to reperfusion-related injury in these patients..$^{14}$ The emergence of technologies such as high-resolution contrast-enhanced vessel wall (VW)-MRI has recently allowed researchers to identify posttreatment wall thickening and enhancement

ABBREVIATIONS ICA = internal carotid artery; MCA = middle cerebral artery; NIHSS = National Institutes of Health Stroke Scale; VW = vessel wall. 
in intracranial arteries in comparison with the contralateral intracranial vasculature as a reference standard. ${ }^{6-9,11-13}$ Using VW-MRI, Kidwell et al. identified posttreatment VW thickening and enhancement in patients who had undergone mechanical thrombectomy and observed that the enhancement was more pronounced than that in patients who had undergone medical therapy alone. ${ }^{6}$ Our objective in the present study was to demonstrate that VW injuries can be detected with VW-MRI and can be used to assess endothelial damage in patients who have undergone various types of thrombectomy approaches.

\section{Methods}

\section{Study Design and Inclusion Criteria}

In this institutional review board-approved cross-sectional study, we enrolled 6 consecutive patients undergoing mechanical thrombectomy for acute ischemic stroke due to large-vessel occlusion and 5 healthy control subjects (that is, without any major medical illness, comorbidities, or vascular disorders). Patients were offered mechanical thrombectomy in accordance with criteria established in a recent randomized controlled trial. ${ }^{1}$ Specifically, thrombectomy patients were included in this study if they had a confirmed proximal anterior cerebral circulation largevessel occlusion, were 18-85 years of age, and underwent the intervention within 6 hours of stroke symptom onset (National Institutes of Health Stroke Scale [NIHSS] Score $>7$ ). Subjects included in the control group were 18-50 years old and were undergoing MRI of the brain, head, neck, or cervical spine for nonvascular indications. Patients with a diagnosis of brain tumor, intracranial atherosclerotic disease, vasculitis, or other CNS inflammatory disorders were excluded. Vessel wall MR images were acquired from the mechanical thrombectomy group at 24 hours after thrombectomy. The control group underwent VW-MRI at the time of MRI for nonvascular indications.

\section{Vessel Wall-MRI Protocol}

Magnetic resonance imaging evaluation of the VW for both the control and mechanical thrombectomy groups was performed using a Signa HDx 3.0-T scanner with an 8-channel head coil (GE Healthcare). The VW protocol included time-of-flight MR angiography of the circle of Willis and a T1-weighted Cube (GE Healthcare) sequence (3D fast spin-echo T1-weighted black-blood sequence, FOV 22 $\mathrm{cm}$, acquired matrix $256 \times 256$, slice thickness $0.8 \mathrm{~mm}$ ) before and after intravenous administration of gadolinium. Vessel wall sequences were acquired volumetrically through the entire circle of Willis. Axial, coronal, and sagittal plane minimum intensity projection (minMIP) reconstructions were performed to evaluate each segment of the targeted arteries. Parenchymal imaging was also performed, including diffusion-weighted, susceptibility weighted, and T2-weighted FLAIR images.

\section{Image Interpretation}

Two independent board-certified neuroradiologists, who were blinded to vessel instrumentation and clinical data, categorized the lumen of each vessel segment as patent or not patent. Then, using pre- and postcontrast T1- weighted VW sequences, they evaluated each vessel segment for T1 hyperintensity and T1 shortening within the arterial wall, which served as evidence of vessel damage and breakdown of the blood-brain barrier. From this assessment, arterial wall enhancement in each segment was defined as present or absent by using the contralateral arteries as a reference standard. Categorization was qualitative and based on clinical experience interpreting a wide range of intracranial VW-MRI studies. The primary end point was VW enhancement. The vessel diameter was measured in each segment $5 \mathrm{~mm}$ beyond the vessel bifurcation to avoid infundibular inclusion. Additional covariates were age, NIHSS score, level of occlusion, stroke etiology, devices used, number of passes required for thrombectomy, Thrombolysis in Cerebral Infarction (TICI) reperfusion score, stroke volume, and 90-day modified Rankin Scale score.

\section{Results}

Postcontrast T1-weighted VW enhancement was detected in $100 \%(6 / 6)$ of the patients undergoing MRI following stent retriever thrombectomy and in $0 \%(0 / 5)$ of the patients undergoing MRI for conditions thought not to contribute to $\mathrm{VW}$ damage (Fig. 1). Subgroup analysis revealed VW enhancement in the $\mathrm{M}_{2}$ segment in $100 \%$ of the thrombectomy patients, in the $\mathrm{M}_{1}$ segment in $83 \%$, and in the internal carotid artery (ICA) in 50\%. One patient demonstrated $\mathrm{A}_{1}$ segment enhancement and was prospectively identified by both radiologists as having undergone anterior cerebral artery thrombectomy due to an embolism during middle cerebral artery (MCA) thrombectomy (Fig. 2). The VW diameters in the thrombectomy patients prior to intervention are recorded in Tables 1 and 2 .

\section{Discussion}

Multiple randomized controlled trials have demonstrated the substantive clinical benefit of mechanical thrombectomy in acute ischemic stroke such that endovascular therapy has become the standard of care in large-vessel occlusion. Though the benefits of mechanical thrombectomy are well established, a subset of patients have poor clinical outcomes despite complete angiographic revascularization after treatment. One potential rationale for the observed clinical and angiographic decoupling pertains
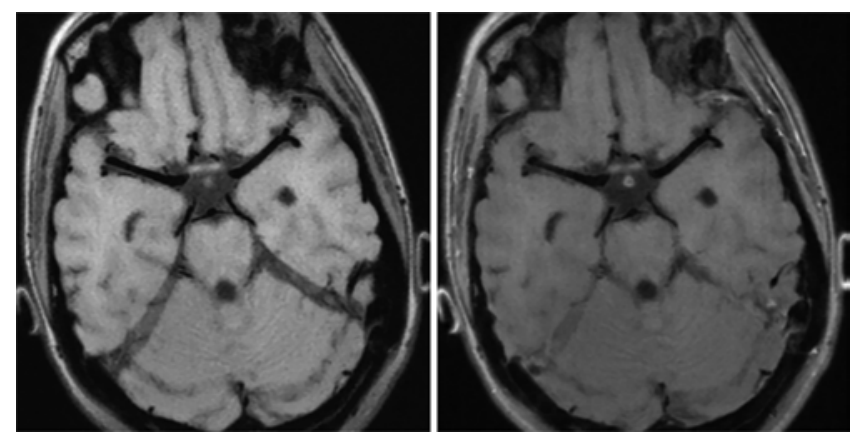

FIG. 1. Axial VW-MR images obtained in a control patient. Left: Postcontrast image showing no evidence of vessel wall enhancement. Right: Postcontrast image demonstrating no enhancement of the wall of the $\mathrm{M}_{1}$ segment. 

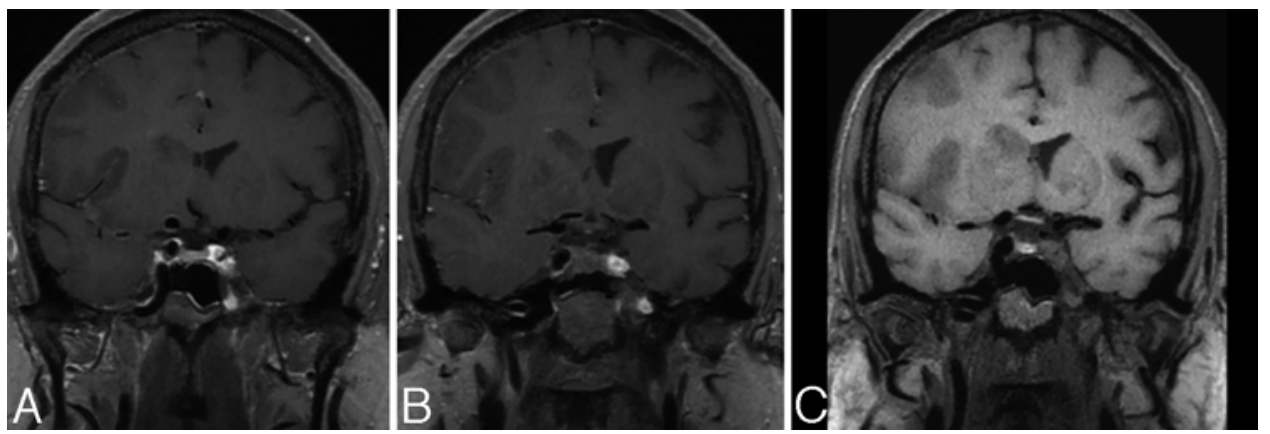

FIG. 2. Coronal VW-MR images obtained following a right MCA mechanical thrombectomy with embolism requiring anterior cerebral artery thrombectomy. Postcontrast images (A and B) demonstrate enhancement of the wall of the right supraclinoid ICA, $A_{1}$ segment, and $M_{1}$ segment compared with their appearance on the precontrast image $(C)$. There is also incidental intrinsic $T 1$ signal in the extracranial left ICA that was occluded at baseline. No enhancement is seen in the contralateral left MCA or intracranial left ICA.

to the mechanical endothelial injury caused by the stent retriever. Mechanical thrombectomy involves the exertion of abrasive force by the device on the endothelium. Therefore, some degree of endothelial injury is expected. Our results demonstrate postcontrast enhancement in the distribution of the mechanical thrombectomy pass that is consistent with endothelial injury. Previous noncontrast studies of VW injury incurred during stent thrombectomy have suggested that clinically relevant VW damage rarely occurs. ${ }^{5}$ Our findings indicate that these negative findings were limited by technique, including a lack of contrastenhanced sequences. Our study demonstrates that vessel injury can be consistently detected utilizing contrast-enhanced 3-T VW-MRI. Additionally, our results demonstrate that endothelial injury is inversely proportional to vessel diameter, which we have previously demonstrated in vitro and in a porcine model. ${ }^{14}$

While the correlation between postthrombectomy VWMRI changes and patient outcomes is not yet fully characterized, we believe that the ability to detect MRI signal changes represents an important and powerful diagnostic stratification method for future studies of mechanical thrombectomy outcomes. Several mechanisms have been proposed to explain how MRI signal enhancement might affect patient outcomes following mechanical thrombectomy: endothelial injury may compromise blood-brain barrier integrity, promote an inflammatory cytokine milieu, compromise downstream cerebral autoregulation, and reduce end-organ tolerance to reperfusion injury.

TABLE 1. Vessel wall diameters in thrombectomy patients prior to intervention

\begin{tabular}{ccccccc}
\hline Case No. & Lt ICA & Lt M $_{1}$ & Lt M $_{2}$ & Rt ICA & Rt M & Rt M \\
\hline 1 & 4.6 & 3.2 & 2.8 & 4.6 & 3.4 & 2.6 \\
\hline 2 & 4.1 & 3.4 & 2.5 & 4.5 & 3.5 & 2.9 \\
\hline 3 & 4.7 & 3.4 & 2.7 & 4.6 & 3.3 & 2.6 \\
\hline 4 & 4.6 & 3.5 & 2.8 & 4.6 & 3.3 & 2.9 \\
\hline 5 & 4.4 & 3.2 & 2.4 & 4.3 & 3.1 & 2.5 \\
\hline 6 & 4.3 & 3.2 & 2.9 & 4.4 & 3.3 & 2.9 \\
\hline
\end{tabular}

Values expressed in $\mathrm{mm}$.
Our study is limited by its small sample size and lack of comparative thrombectomy techniques, and it is not powered to evaluate downstream end-organ and clinical effects. Having now established the presence of previously undetected in vivo injury with thrombectomy, subsequent study may correlate in vivo endothelial damage detected by MRI with the downstream end-organ effects. Moreover, future research will compare different thrombectomy techniques including aspiration for differential patterns of endothelial trauma, as established by our group in artificial vessel and animal models. ${ }^{14}$ Ultimately, we anticipate that studies of endothelial injury patterns demonstrated by contrast-enhanced VW-MRI will guide the design and sizing of future embolectomy devices. We may further identify a subpopulation of patients appropriate for neuroprotective agent trials or therapeutic targets that blunt the negative consequences of endothelial trauma.

\section{Conclusions}

Our findings demonstrate that VW injuries incurred during stent retriever thrombectomy can be consistently detected utilizing contrast-enhanced 3-T VW-MRI. Furthermore, we observed a greater degree of endothelial injury when the thrombectomy device was oversized relative to the target vessel, indicating the importance of judicious device selection. Our pilot study represents a successful effort to characterize VW biology in vivo with high fidelity, based on endovascular instrumentation. However, further studies are needed to evaluate the differential effects of thrombectomy devices and techniques on the VW endothelium, to characterize the histological changes incurred, and to correlate the effects of VW damage with clinical outcomes.

TABLE 2. Mean vessel wall diameters in thrombectomy patients prior to intervention

\begin{tabular}{clcc}
\hline Parameter & \multicolumn{1}{c}{ ICA } & \multicolumn{1}{c}{$\mathrm{M}_{1}$} & $\mathrm{M}_{2}$ \\
\hline Mean & 4.475 & 3.333333 & 2.708333 \\
\hline SD & 0.17645499 & 0.132916 & 0.183196 \\
\hline
\end{tabular}

Values expressed in $\mathrm{mm}$. 


\section{Acknowledgments}

This study was supported by an NIH Short-Term Research Training Grant (No. 5T35HL007491-35).

\section{References}

1. Berkhemer OA, Fransen PSS, Beumer D, van den Berg LA, Lingsma HF, Yoo AJ, et al: A randomized trial of intraarterial treatment for acute ischemic stroke. N Engl J Med 372:11-20, 2015

2. Butler PJ, Norwich G, Weinbaum S, Chien S: Shear stress induces a time- and position-dependent increase in endothelial cell membrane fluidity. Am J Physiol Cell Physiol 280:C962-C969, 2001

3. Fransen PSS, Beumer D, Berkhemer OA, van den Berg LA, Lingsma $\mathrm{H}$, van der Lugt A, et al: MR CLEAN, a multicenter randomized clinical trial of endovascular treatment for acute ischemic stroke in the Netherlands: study protocol for a randomized controlled trial. Trials 15:343, 2014

4. Galbraith CG, Skalak R, Chien S: Shear stress induces spatial reorganization of the endothelial cell cytoskeleton. Cell Motil Cytoskeleton 40:317-330, 1998

5. Hsieh K, Verma RK, Schroth G, Gratz PP, Kellner-Weldon F, Gralla J, et al: Multimodal 3 Tesla MRI confirms intact arterial wall in acute stroke patients after stent-retriever thrombectomy. Stroke 45:3430-3432, 2014

6. Kidwell CS, Jahan R, Gornbein J, Alger JR, Nenov V, Ajani $\mathrm{Z}$, et al: A trial of imaging selection and endovascular treatment for ischemic stroke. N Engl J Med 368:914-923, 2013

7. Klein IF, Lavallée PC, Touboul PJ, Schouman-Claeys E, Amarenco P: In vivo middle cerebral artery plaque imaging by high-resolution MRI. Neurology 67:327-329, 2006

8. Küker W, Gaertner S, Nagele T, Dopfer C, Schoning M, Fiehler J, et al: Vessel wall contrast enhancement: a diagnostic sign of cerebral vasculitis. Cerebrovasc Dis 26:23-29, 2008

9. Mandell DM, Matouk CC, Farb RI, Krings T, Agid R, terBrugge K, et al: Vessel wall MRI to differentiate between reversible cerebral vasoconstriction syndrome and central nervous system vasculitis: preliminary results. Stroke 43:860-862, 2012
10. Pan J, Konstas AA, Bateman B, Ortolano GA, Pile-Spellman $\mathrm{J}$ : Reperfusion injury following cerebral ischemia: pathophysiology, MR imaging, and potential therapies. Neuroradiology 49:93-102, 2007

11. Power S, Matouk C, Casaubon LK, Silver FL, Krings T, Mikulis DJ, et al: Vessel wall magnetic resonance imaging in acute ischemic stroke: effects of embolism and mechanical thrombectomy on the arterial wall. Stroke 45:2330-2334, 2014

12. Qiao Y, Steinman DA, Qin Q, Etesami M, Schär M, Astor $\mathrm{BC}$, et al: Intracranial arterial wall imaging using threedimensional high isotropic resolution black blood MRI at 3.0 Tesla. J Magn Reson Imaging 34:22-30, 2011

13. Swartz RH, Bhuta SS, Farb RI, Agid R, Willinsky RA, terBrugge KG, et al: Intracranial arterial wall imaging using high-resolution 3-tesla contrast-enhanced MRI. Neurology 72:627-634, 2009

14. Teng D, Pannell JS, Rennert RC, Li J, Li YS, Wong VW, et al: Endothelial trauma from mechanical thrombectomy in acute stroke: in vitro live-cell platform with animal validation. Stroke 46:1099-1106, 2015

\section{Disclosures}

Dr. Khalessi is a consultant for Medtronic. Dr. Pannell is a consultant for Stryker.

\section{Author Contributions}

Conception and design: Khalessi, Abraham, Pannell, Rennert, Lee. Acquisition of data: Abraham, Pannell, Wali, Lee. Analysis and interpretation of data: Abraham, Pannell. Drafting the article: Abraham. Critically revising the article: all authors. Reviewed submitted version of manuscript: all authors. Administrative/ technical/material support: Santiago-Dieppa. Study supervision: Khalessi, Pannell.

\section{Correspondence}

Alexander A. Khalessi, Department of Neurosurgery, University of California, San Diego, 200 West Arbor Dr., San Diego, CA 92103. email: akhalessi@ucsd.edu. 